\title{
Holography, Entropy and Extra Dimensions
}

\author{
Deog Ki Hong* \\ Department of Physics \\ Pusan National University, Pusan 609-735, Korea \\ Stephen D.H. Hsu ${ }^{\dagger}$ \\ Department of Physics \\ University of Oregon, Eugene OR 97403-5203
}

December 13, 2018

\begin{abstract}
We show that higher dimensional models (brane worlds) in which the scale of quantum gravity $M_{*}$ is much smaller than the apparent scale $M_{P} \sim 10^{19} \mathrm{GeV}$ violate the covariant entropy bound arising from holography. The thermodynamic entropies of astrophysical black holes and sub-horizon volumes during big bang nucleosynthesis exceed the relevant bounds unless $M_{*}>10^{(4-6)} \mathrm{TeV}$, so a hierarchy relative to the weak scale is unavoidable. We discuss the implications for extra dimensions as well as holography.
\end{abstract}

*dkhong@pnu.edu

†hsu@duende.uoregon.edu 
Recent progress in string theory and the study of quantum black holes strongly suggests that the maximum information content of a spacetime region is related to its surface area $[1,2,3]$. The idea has its origins in the proposal of Bekenstein that the area of a black hole is proportional to its entropy [4], and that black holes obey a generalized second law of thermodynamics (GSL) [5]. A covariant generalization of these ideas [6, 7] has passed a number of theoretical tests, and implies a deep relationship between geometry and information which arises due to quantum gravity.

At first glance, the proposal that the maximum information in a volume only grows as the surface area seems obviously false. At least, it contradicts the usual counting used in statistical physics or quantum field theory. However, 't Hooft [1] showed that if configurations in the volume which would have already led to gravitational collapse are excluded, the number of states grows less rapidly than the exponential of the area, or equivalently that the entropy is bounded above by the area, in Planck units. We give a version of 't Hooft's argument below, generalized to D spacetime dimensions.

Models with $D>4$ dimensions, in which ordinary matter is constrained to a $3+1$ dimensional subspace (the brane), while gravity propagates in all D dimensions, allow for the possibility that the fundamental scale of gravity, $M_{*}$, is much smaller than the apparent Planck scale, $M_{P}=10^{19} \mathrm{GeV}$, governing gravitational interactions on the 3-brane [8, 9]. If $M_{*} \sim \mathrm{TeV}$, these models solve the hierarchy problem in a novel manner. However, the surface entropy or information density is consequently much smaller than in ordinary theories of gravity. We refer to models in which the extra dimensional space is flat as ADD models [8], and those with warped extra dimensions as RS models [9].

In this letter we examine systems, such as supernova cores and the early universe, which are accurately described by ordinary thermodynamics. In order that these systems not saturate holographic bounds on their entropy, we deduce a lower bound on the fundamental scale of quantum gravity: $M_{*}>10^{(4-6)} \mathrm{TeV}$. Clearly, this bound is problematic for brane world solutions to the hierarchy problem.

\section{Notation and Preliminaries}

$M_{*}$ is the dynamical scale of quantum gravity. In ADD/RS universes it can be as low as a few TeV. $M_{P}$ is the apparent Planck scale, or $10^{19} \mathrm{GeV}$. $D$ is the number of spacetime dimensions. In $D$ dimensions the surface area (or surface volume, for $D>4$ ) of a black hole scales like $R^{D-2}$.

The Einstein action is

$$
S=M_{*}^{D-2} \int d^{D} x \sqrt{-g} \mathcal{R}
$$


and the (dimensionless) gravitational potential per unit test mass behaves as

$$
\Phi \sim \frac{E}{M_{*}^{D-2} R^{D-3}} \sim \frac{E R}{\left(M_{*} R\right)^{D-2}}
$$

Thus, the Schwarzschild radius in $D$ dimensions is:

$$
R_{s} \sim\left(M_{*}^{2-D} E\right)^{1 /(D-3)}
$$

\section{Holography in D dimensions}

To generalize 't Hooft's result [1], we compute the entropy of a region of size $R$ under the condition that it is on the verge of collapsing to a black hole, or $R \sim R_{s}$. We assume a roughly spherical geometry throughout. As we will see later in the paper, assuming a brane world geometry modifies the results substantially.

The dominant configurations are thermal ${ }^{1}$, characterized by a temperature $T$, energy density $T^{D}$ and entropy density $T^{D-1}$. The total energy and entropy of the region are

$$
E \sim R^{D-1} T^{D}, \quad S \sim R^{D-1} T^{D-1}
$$

Substituting (3) into (4), we obtain the bound:

$$
M_{*}^{2-D} R^{D-1} T^{D}<R^{D-3}
$$

which implies

$$
T<\left(M_{*}^{D-2} R^{-2}\right)^{1 / D}
$$

and the entropy bound

$$
S \sim R^{D-1} T^{D-1}<M_{*}^{(D-2)(D-1) / D} R^{D-1} R^{-2(D-1) / D}
$$

or

$$
S<R^{D-3+2 / D} \sim R^{(D-2)+(2 / D-1)}
$$

in appropriate units. This is always at least as strong as the holographic bound, which depends on the surface area: $S<R^{D-2}$. The two bounds coincide when $D=2$. Note that in $D=2$ the boundary of the black hole is simply two points, so our bound should scale as $R^{0}$, which it does. For $D=4$ we obtain 't Hooft's result [1] that $S<A^{3 / 4}$. When we allow for an admixture of black holes in the region, the holographic bound is recovered. (It is saturated when the region becomes a single black hole.)

\footnotetext{
${ }^{1}$ Temperature here is fictitious. It lets us characterize the dominant configurations in phase space, since in thermal equilibrium the entropy density is maximal for a given energy density.
} 
Bousso [7, 3] formulated a covariant generalization to previous holographic entropy bounds which addresses some of the failings of the original spacelike bounds and which applies even in cosmological or strongly gravitating settings. In what follows we will apply Bousso's covariant entropy bound (CB) to higher dimensional theories. The bound can be stated as follows.

Let $A(B)$ be the area of an arbitrary $D-2$ dimensional spatial surface, which need not be closed. A D-1 dimensional hypersurface $L$ is the light-sheet of $B$ if $L$ is generated by light rays extending orthogonally from $B$, which have non-positive expansion everywhere on $L$. Let $S$ be the entropy on any light sheet of $B$. Then $S \leq \frac{1}{4} A(B)$.

The entropy of a light sheet is given by that of the matter intersecting the sheet. For simple cases, such as a suitable closed spacelike surface surrounding a weakly gravitating system, the covariant bound reduces to the usual area bound.

\section{Application to extra dimensions}

Assume an ADD/RS world in which the standard model degrees of freedom are confined to a 3-brane while the gravitational degrees of freedom propagate in $D>4$ dimensions. The large effective volume $V_{w}$ of the bulk allows the apparent Planck scale $M_{P}$ to be much larger than the true dynamical scale of gravity $M_{*} \sim \mathrm{TeV}$. Consider a spacelike region $\mathrm{V}$ of extent $r$ on the 3-brane and thickness $l$ in the orthogonal extra dimensions. The boundary of $\mathrm{V}$ consists of components whose surface areas scale as $r^{3} l^{(D-5)}$ and $r^{2} l^{(D-4)}$. The first surface component is obtained by setting the extra-dimensional coordinates at their extreme (boundary) values and allowing the coordinates $\left\{x_{1-3}\right\}$ to vary throughout the intersection of $\mathrm{V}$ with the 3 -brane. The second is obtained by setting $\left\{x_{1-3}\right\}$ at their extreme values (i.e., the boundary on the 3-brane) and letting the extra-dimensional coordinates to vary over a range of size $l$.

We will apply the covariant bound to a hypersurface B which corresponds to the second part of the boundary of $\mathrm{V}$, the one whose area scales as $r^{2} l^{(D-4)}$. The light sheet $\mathrm{L}$ associated with $\mathrm{B}$ intersects all of the ordinary matter in $\mathrm{V}$ because, by assumption, it is confined on the brane ${ }^{2}$. Therefore, the entropy on $\mathrm{L}$ is simply that of the ordinary matter in $\mathrm{V}$.

Let $\mathrm{V}$ have the same shape as the brane, with thickness 1 of order $M_{*}^{-1}$, so that its surface area is of order $r^{2}$ in units of $M_{*}$. (It is possible that the brane is thicker than $M_{*}^{-1}$, forcing us to use a larger hypersurface with more entropy density, however it is hard to imagine that the brane thickness is parametrically larger than the fundamental length scale.)

\footnotetext{
${ }^{2}$ If the matter were not confined on the brane, we would have to include the first surface area component (the $r^{3} l^{(D-5)}$ component) in B to obtain a bound on the matter entropy.
} 


\section{The covariant bound is violated during the big bang.}

Let the temperature in the early universe at temperature T. Impose that the fiducial region $\mathrm{V}$ saturate the holographic bound, so $r$ satisfies

$$
T^{3} r^{3} \sim M_{*}^{2} r^{2}
$$

or

$$
r \sim T^{-1}\left(\frac{M_{*}}{T}\right)^{2}
$$

Now compare our fiducial volume to a cosmological horizon volume of size $d_{H} \sim M_{P} / T^{2}$ (assuming radiation domination). The ratio of $r$ to $d_{H}$ is

$$
\frac{d_{H}}{r} \sim \frac{T}{M_{*}} \frac{M_{P}}{M_{*}} \sim \frac{T}{10^{-4} \mathrm{eV}}
$$

For the matter-dominant epoch, the horizon distance is given as $d_{H} \sim\left(M_{P} / T_{d}^{2}\right)\left(T_{d} / T\right)^{3 / 2}$, where $T_{d} \simeq 10 \mathrm{eV}$ is the onset temperature of matter domination. The ratio then becomes

$$
\frac{d_{H}}{r} \sim \frac{M_{P}}{M_{*}}\left(\frac{T^{3}}{M_{*}^{2} T_{d}}\right)^{1 / 2} \sim\left(\frac{T}{10^{-2} \mathrm{eV}}\right)^{3 / 2} .
$$

We find that for any temperature higher than $10^{-2} \mathrm{eV}$ the causal horizon contains more degrees of freedom than are allowed according to the $\mathrm{CB}$ applied to the fundamental theory.

Our understanding of thermodynamics and statistical physics is based on counting states. If the $\mathrm{CB}$ is correct, the early universe in the brane worlds under consideration will likely not obey the usual laws of thermal physics at temperatures $>10^{-2} \mathrm{eV}$. This makes our understanding of nucleosynthesis and the microwave background problematic.

In order that our thermodynamic description of nucleosynthesis (at $T \sim 10 \mathrm{MeV}$ ) not be invalidated by holography, we find that $M_{*}>10^{4} \mathrm{TeV}$. (This bound is reduced slightly from (11) when prefactors in the expressions for the entropy density and horizon size are included.)

The maximum violation of the covariant entropy bound can be deduced by considering a thermal region of temperature $T$ and extent $r \sim M_{P} / T^{2}$ on the 3-brane (the largest it could be and still fit in a cosmological horizon). Comparing its entropy $S \sim r^{3} T^{3} \sim\left(M_{P} / T\right)^{3}$ to its surface area $r^{2}$, and taking the maximum temperature $T \sim M_{*}$, we see that the entropy bound can be violated by a factor of $\left(M_{P} / M_{*}\right) \sim 10^{16}$ for $\mathrm{TeV}$ gravity.

\section{The covariant bound is violated by supernova cores.}

Consider the supernova of a star of mass $M>8 M_{\odot}$, which is powered by the collapse of an iron core and leads to neutron star or black hole formation. In this process the entropy of 
the collapsed neutron star is of order one per nucleon, so the total entropy is roughly $10^{57}$. The radius of the core is a few to ten kilometers, so that its area $\left(10^{12} \mathrm{~cm}^{2}\right)$ in $M_{*}$ units is only $10^{46}$, where again we take a fiducial volume of thickness just greater than that of the brane. (As in the cosmological case the degrees of freedom we are counting are all confined to the brane.) Unless $M_{*}>10^{6} \mathrm{TeV}$ there is a conflict between the usual thermodynamic description of supernova collapse and the holographic entropy bound.

\section{Black hole entropy bound vs. covariant bound}

Susskind [2] imagines a process in which a thermodynamic system is converted into a black hole by collapsing a spherical shell around it. Using the GSL, one obtains a bound on the entropy of the system: $S_{\text {matter }} \leq A / 4$, where $A$ is the area of the black hole formed. This is a weaker conjecture than the covariant bound, and has considerable theoretical support $[2,3,4,5]$. In the application of the $\mathrm{CB}$ we are free to choose the hypersurface $\mathrm{B}$, as long as its lightsheet intersects all of the matter whose entropy we wish to bound, whereas in the black hole bound the area which appears is that of the black hole which is formed. The black hole entropy bound is sensitive to the dynamics of horizon formation.

In TeV gravity scenarios, the black hole size on the 3-brane is controlled by the apparent Planck scale $M_{P}=10^{19} \mathrm{GeV}$. The extent of the horizon in the perpendicular directions off the brane depends on the model, unless the hole is very small.

In ADD worlds, the horizon of an astrophysical black hole likely extends to the boundary of the compact extra dimensions. As discussed in [10], large black holes have geometry $S^{2} \times T^{D-4}$, and the horizon includes all of the extra volume $V_{w}$. Due to this additional extra-dimensional volume, the resulting entropy density is the same as in $3+1$ dimensions and there is no obvious violation of any bounds.

In RS scenarios, however, black holes are confined to the brane and have a pancake-like geometry $[11,12]$. The black hole size in the direction transverse to the brane grows only logarithmically with the mass $M$. Thus far, no one has computed the Hawking temperature or entropy of a pancake black hole. In fact, exact solutions describing these objects have yet to be obtained. Let us assume, motivated by holography, that the entropy of a pancake black hole continues to be of order its surface area in units of $M_{*}$. The surface area of a large hole is dominated by the $r^{3} l^{D-5}$ component, so the black hole entropy bound arising from the Susskind construction in RS worlds is of the form

$$
S<\left(r M_{*}\right)^{3}
$$

That is, the upper bound on the entropy grows with the apparent 3-volume of the region. In this case the black hole bound is clearly weaker than the covariant bound, because the 
surface B used in the application of the latter is much smaller than the area of the pancake hole. Interestingly, (13) is the same result one would have obtained naively from $D=4$ quantum field theory in the absence of gravity, with ultraviolet cutoff $M_{*}$ !

Assuming that the entropy of a pancake black hole is given by its area in $M_{*}$ units, the relationship between entropy and temperature implies that the Hawking temperature is greatly enhanced:

$$
T=\frac{d M}{d S} \sim \frac{1}{r}\left(\frac{M_{P}}{M}\right)\left(\frac{M_{P}}{M_{*}}\right)^{3} .
$$

For example, a solar mass black hole would have temperature $T \sim \mathrm{eV}$ if $M_{*} \sim \mathrm{TeV}$. However, (14) should be treated with caution. The calculation of the Hawking temperature for a pancake black hole is rather confusing - an observer on the brane would seem to obtain the usual result, while an observer in the normal direction might find a different temperature in radiated gravitons, depending on the precise (currently unknown) nature of the horizon in the normal coordinate.

\section{Discussion}

Our results can be interpreted in two ways, depending on how one views holography and related entropy bounds.

It seems likely that holography is a deep result of quantum gravity, relating geometry and information in a new way [3]. If so, it provides important constraints on extra dimensional models. Our analysis shows that the ordinary thermodynamic treatment of nucleosynthesis and supernovae are in conflict with the covariant bound. In other words, brane worlds obeying holography do not reproduce the observed big bang thermal evolution or stellar collapse. Exactly what replaces the usual behavior is unclear - presumably it is highly nonlocal - but the number of degrees of freedom is drastically less than in the thermodynamic description.

An alternative point of view is to regard brane worlds as a challenge to holography. If such worlds exist they have the potential to violate the entropic bounds by arbitrarily large factors. However, it must be noted that the basic dynamical assumptions underlying the scenarios (that the 3-brane and bulk geometry arise as a ground state of quantum gravity) have never been justified. All violations discussed here require a hierarchy between $M_{P}$ and $M_{*}$, or equivalently that the extra-dimensional volume factor $V_{w}=\int d^{D-4} x \sqrt{-g_{(D-4)}}$ exceed its "natural" size $\sim M_{*}^{-(D-4)}$.

Finally, we note that the brane, or whatever confines matter to 3 spatial dimensions, is absolutely necessary for these entropy violations. Without the brane, matter initially in a region with small extent in the extra $(D-4)$ dimensions will inevitably spread out due to 
the uncertainty principle. For ordinary matter in classical general relativity, in the absence of branes, Wald and collaborators [13] have proven the covariant entropy bound subject to some technical assumptions.

\section{Acknowledgements}

The work of D.K.H. is supported by KRF PBRG 2002-070-C00022. The work of S.H. was supported in part under DOE contract DE-FG06-85ER40224 and by the NSF through through the USA-Korea Cooperative Science Program, 9982164.

\section{References}

[1] G. 't Hooft, "Dimensional Reduction In Quantum Gravity," edited by A. Ali, J. Ellis, and S. Randjbar-Daemi, Salamfestschrift: A Collection of Talks (World Scientific, Singapore,1993), arXiv:gr-qc/9310026.

[2] L. Susskind, J. Math. Phys. 36 (1995) 6377 [arXiv:hep-th/9409089].

[3] R. Bousso, Rev. Mod. Phys. 74 (2002) 825 [arXiv:hep-th/0203101].

[4] J. D. Bekenstein, Phys. Rev. D 7 (1973) 2333; S. W. Hawking, Commun. Math. Phys. 43 (1975) 199; Phys. Rev. D 13 (1976) 191.

[5] J. D. Bekenstein, Phys. Rev. D 9 (1974) 3292; 23 (1981) 287; 49 (1994) 1912 [arXiv:grqc/9307035].

[6] W. Fischler and L. Susskind, arXiv:hep-th/9806039.

[7] R. Bousso, JHEP 9907 (1999) 004 [arXiv:hep-th/9905177]; Phys. Rev. Lett. 90 (2003) 121302 [arXiv:hep-th/0210295].

[8] N. Arkani-Hamed, S. Dimopoulos and G. R. Dvali, Phys. Lett. B 429 (1998) 263 [arXiv:hep-ph/9803315]; Phys. Rev. D 59 (1999) 086004 [arXiv:hep-ph/9807344]; I. Antoniadis, N. Arkani-Hamed, S. Dimopoulos and G. R. Dvali, Phys. Lett. B 436, 257 (1998) [arXiv:hep-ph/9804398].

[9] L. Randall and R. Sundrum, Phys. Rev. Lett. 83 (1999) 3370 [arXiv:hep-ph/9905221]; 83 (1999) 4690 [arXiv:hep-th/9906064].

[10] P. C. Argyres, S. Dimopoulos and J. March-Russell, Phys. Lett. B 441 (1998) 96 [arXiv:hep-th/9808138]. 
[11] S. B. Giddings, E. Katz and L. Randall, JHEP 0003 (2000) 023 [arXiv:hep-th/0002091].

[12] R. Casadio and L. Mazzacurati, Mod. Phys. Lett. A 18, 651 (2003) [arXiv:grqc/0205129].

[13] E. E. Flanagan, D. Marolf and R. M. Wald, Phys. Rev. D 62 (2000) 084035 [arXiv:hepth/9908070]. 